\title{
Modelling of the Temporal and Spatial Evolutions of Weakly Nonlinear Random Directional Waves with the Modified Nonlinear Schrödinger
}

\section{Equations}

\author{
H.D. Zhang ${ }^{1}$, C. Guedes Soares ${ }^{1}$, M. Onorato ${ }^{2}$, A. Toffoli ${ }^{3}$ \\ ${ }^{1}$ Centre for Marine Technology and Ocean Engineering (CENTEC), Instituto Superior
} Técnico, University of Lisbon, Portugal

${ }^{2}$ Dipartimento di Fisica, Universitá di Torino, Via P. Giuria 1, 10125 Torino, Italy

${ }^{3}$ Centre for Ocean Engineering Science and Technology, Swinburne University of Technology, P.O. Box 218, Hawthorn, Victoria 3122, Australia

Correspondence to: C. Guedes Soares (c.guedes.soares@ centec.tecnico.ulisboa.pt)

\begin{abstract}
The temporal and spatial evolutions of nonlinear wave group with an initial Gaussian envelope are theoretically studied under the governing of MNLS equations, demonstrating that the temporal and spatial versions of numerical model are not always consistent in the whole evolution process, particularly in the presence of strong nonlinearity. Moreover, a large set of numerical simulations, performed respectively by these two versions of numerical model, are systematically compared to mechanically generated waves with different initial directional spreading and Benjamin-Feir Index, mainly focusing on the evolution properties of surface elevations such as the coefficients of skewness and kurtosis, the probability density function, and the maximal surface elevation. On the whole, it can be argued that the statistical
\end{abstract}


properties of both numerically simulated wave fields are basically consistent with the laboratory observations.

Keywords: Benjamin-Feir Index; Coefficients of skewness and kurtosis; Directional spreading; Nonlinear instability; MNLS equation; Temporal and spatial Evolutions; Surface gravity waves

\section{Introduction}

Relatively rare and unusually large water waves, often called freak, rogue or abnormal waves, have been given a lot of attention by scientists and engineers in the past decades. The immediate interest and motivation for studying such waves arise from their catastrophic damage on floating offshore structures and ships which has been recorded in many reports [1].

The mechanisms of abnormal or freak wave generation are various and complicated. Besides the linear superposition of Fourier components with coherent phases and strong wave-current interaction or wave diffraction, it has been established that the nonlinear dispersive focusing or modulational instability can explain many of the features of the dynamics of abnormal waves as reviewed by Kharif and Pelinovsky [2]. This instability was first discovered by Lighthill [3] while the theory was developed independently by Benjamin and Feir [4] as well as Zakharov [5].

The simplest nonlinear model that contains the representation of Benjamin-Feir instability is the cubic nonlinear Schrödinger equation which can be derived from the Euler equations by assuming potential flow of free-surface waves that are weakly nonlinear and narrow-banded. Comparison of the numerical simulations based on NLS equation with experiments indicates 
that NLS equation is adequate enough for qualitative description of the global properties of the envelope evolution such as focusing of water waves observed in deepwater condition, but it is incapable of capturing more subtle features, for example, the emerging front-tail asymmetry observed in the experiments [6] [7]. Hence, a more advanced model, which is a higher-order extension of the NLS equation, was derived [8] on the basis of a systematic asymptotic procedure. The similar expression can also be obtained from the third-order Zakharov integral equation by adding the assumption of narrow-band spectral width [9]. In order to expand the application of NLS-type equations, further modification on the bandwidth constraint was made by Trulsen and Dysthe [10] and Trulsen et al. [11].

Generally speaking, in laboratory tests, wave data are accumulated using sensors located at fixed positions in the wave basin, thus recording the temporal variation of water surface elevation. To perform quantitative comparison with the numerical model prediction, the governing equation has to be modified to a spatial form such as what was done by Lo and Mei [12]. As expected, this spatial version of Dysthe equation can be derived from the spatial form of Zakharov equation as well [13].

For the purpose of describing more broad-banded sea states and gaining more accurate results, the aforementioned Zakharov equation may also work as an alternative numerical model (see e.g. [14]). Furthermore, the surface elevation can be simulated directly from the potential Euler equations as well. In this respect, there exist a lot of methods that allow to simulation of fully nonlinear waves (e.g., [15]-[17]). However, due to the computational burden, the truncated Euler equations are often calculated with a higher-order spectral method, which was derived independently by Dommermuth and Yue [18] and West et al. 
[19]. Recently, it is revealed that results of this method are almost consistent with those of simpler model of NLS-type equations in directional sea states [20] [21].

Therefore, in this study the modified nonlinear Schrödinger equations including spatial and temporal forms are used as the governing equations to research the directional wave field considering that they are much simpler and computationally cheaper. Moreover, these models are particularly suitable to investigate wave statistics, which requires the calculation of many realizations of a random sea surface [22] [23]. Besides, another very effective method to investigate the abnormal waves is the quasi-determinism (QD) theory of Boccotti [24] [25], as demonstrated in the comparison with laboratory generated New Year wave [26].

It is well known that for long-crested waves at third order in wave steepness, wave trains can be unstable to small perturbations which can cause a local exponential growth in the wave amplitude within a time frame of a few tens of wave periods [27]. However, threedimensional numerical simulations governed by the modified NLS equation [22] [28] illustrates that the number of extreme wave events is reduced when the directional spreading of the initial spectrum is increased. For short-crested waves the deviation from Gaussian statistics is only due to bound wave contributions while free waves preserve normal statistics despite third-order nonlinear evolution. On the foundation of a large set of numerical simulations, Gramstad and Trulsen [23] performed a detailed analysis on the effect of directionality, revealing how the occurrence of freak waves in deep water depends on the group and crest lengths for a fixed steepness. The associated experimental investigation was first shown by Stansberg [29], who casted some light on the effects of directionality on the fourth-order moment of the surface elevation. More recent experimental results were reported 
by Waseda [30] and Onorato et al. [31], indicating that the occurrence of extreme waves can be significantly reduced when the directionality broadens.

NLS-type equations have received considerable attention by wave researchers and represent an important source of information for the statistics of extreme waves which are rare in nature. Although various forms of NLS-type equations have been applied in the comparison with measurements in the wave basins and open seas [32]-[35], the research on the numerical difference of the spatial and temporal model is still not enough considering that only some discussions on unidirectional nonlinear wave group with an initial Gaussian envelope were presented by Shemer and Dorfman [36]. Therefore, the main aim in this study is to further check the applicability of spatial and temporal MNLS equations in simulating the more realistic sea states characterized by JONSWAP spectrum with different directionality and Benjamin-Feir Index.

This paper is organized as follows: Section 2 gives a short review on the numerical models and basic statistical theory used in this paper. Section 3 briefly describes the laboratory facilities of Marintek, related experimental setup and corresponding numerical details. Section 4 is devoted to the comparison of temporal and spatial evolutions of Gaussian envelope, as well as the comparative analysis on statistical properties between the observed and simulated directional wave fields. The important conclusions are summarized in the last section.

\section{Theory}

\subsection{Modified nonlinear Schrodinger equations}


Evolution of the wave group can be represented by the variation of complex amplitude of either the surface elevation or the velocity potential at the free surface in time or space domain. In this work, the equations expressed in the non-dimensional complex amplitude of the surface elevation are employed to simulate the evolution of wave field. The exact expressions for the temporal and spatial MNLS equations are given by

$$
\begin{aligned}
B_{t}+ & \frac{1}{2} B_{x}+\frac{\mathrm{i}}{8} B_{x x}-\frac{\mathrm{i}}{4} B_{y y}+\frac{\mathrm{i}}{2}|B|^{2} B-\frac{1}{16} B_{x x x}+\frac{3}{8} B_{x y y} \\
+ & \frac{3}{2}|B|^{2} B_{x}+\frac{1}{4} B^{2} B_{x}^{*}+\mathrm{i} B \bar{\phi}_{x}=0, \quad z=0, \\
B_{x} & +2 B_{t}+\mathrm{i} B_{t t}-\frac{\mathrm{i}}{2} B_{y y}+\mathrm{i}|B|^{2} B-B_{t y y}-8|B|^{2} B_{t} \\
& -2 B^{2} B_{t}^{*}-4 \mathrm{i} B \bar{\phi}_{t}=0, \quad z=0,
\end{aligned}
$$

respectively [20] [22], where the induced mean flow potential $\bar{\phi}$ is governed by

$$
\begin{gathered}
\nabla^{2} \bar{\phi}=0, \quad-h<z<0, \\
\bar{\phi}_{z}=\frac{1}{2}\left(|B|^{2}\right)_{x}=-\left(|B|^{2}\right)_{t}, \quad z=0, \\
\bar{\phi}_{z}=0, \quad z=-h,
\end{gathered}
$$

Here the subscripts denote partial derivatives. Time and length are scaled by peak frequency $\omega_{p}$ and corresponding peak wave number $k_{p}$, respectively. If the free wave complex amplitude $B$ is solved, the surface displacement can be reconstructed by the following formulae:

$$
\eta=\bar{\eta}+\frac{1}{2}\left(B \mathrm{e}^{\mathrm{i}(x-t)}+B_{2} \mathrm{e}^{2 \mathrm{i}(x-t)}+B_{3} \mathrm{e}^{3 \mathrm{i}(x-t)}+\cdots+\text { c.c. }\right)
$$

where c.c. denotes complex conjugate and the bound contributions are given by

$$
\begin{gathered}
\bar{\eta}=\frac{1}{2} \bar{\phi}_{x}=-\bar{\phi}_{t}, \\
B_{2}=\frac{1}{2} B^{2}-\frac{\mathrm{i}}{2} B B_{x}=\frac{1}{2} B^{2}+\mathrm{i} B B_{t}
\end{gathered}
$$




$$
B_{3}=\frac{3}{8} B^{3}
$$

Both $B_{2}$ and $B_{3}$ are phase locked to $B$ and the mean surface elevation $\bar{\eta}$ is associated with the radiation stress.

The split-step Fourier method described in Lo and Mei [12] [37] is used to solve the MNLS equations numerically in a two-dimensional rectangular domain with an imposed periodic boundary condition in both horizontal directions. For example, in the temporal evolution, the envelope function $B(\boldsymbol{x}, t)$ is solved in the modulational wavenumber space $\boldsymbol{K}=$ $\left(K_{x}, K_{y}\right)=\left(\boldsymbol{k}-\boldsymbol{k}_{p}\right) / k_{p}$ and only the modes within $\left|K_{x}\right| \leq 1$ and $\left|K_{y}\right| \leq 1$ are used. The computation domain is $256 L \times 256 L$, over which a uniform grid size $N_{x}=N_{y}=512$ is applied. To resolve the rapidly oscillating surface elevation $\eta(x, t)$, a resampling is performed. For the resolution explained above, the minimum number of points that has to be used to describe the full surface elevation which includes the third-order bound wave effect is $3072 \times 1536$, corresponding to about 12 points per dominant wavelength $L$ downstream the wave tank. The similar setup of these parameters is applied in the spatial evolution as well. Detailed description of the numerical method can be found in the work of Socquet-Juglard et al. [22]. Considering that the free wave envelop $B$ is slowly varying under the narrow-band assumption, the integration step used in the numerical simulation can be chosen relatively large [20] such as $\Delta t=\Delta x=2 \pi / 10$. It corresponds to 10 integration steps per peak period $T$ in the temporal evolution and 10 integration steps per peak wavelength $L$ in the spatial evolution. These parameters can provide a satisfactory accuracy, and the energy in all simulations is verified to be conserved to within $0.4 \%$ of the initial value.

\subsection{Initial random directional wave field}


The initial wave field is specified by the JONSWAP spectrum with a directional spreading function

$$
S(k, \theta)=\frac{\alpha}{2 k^{3}} \exp \left[-\frac{5}{4}\left(\frac{k_{p}}{k}\right)^{2}\right] \gamma^{\exp \left[-\left(\sqrt{k / k_{p}}-1\right)^{2} /\left(2 \sigma_{0}^{2}\right)\right]} D(\theta),
$$

where $k_{p}$ is the peak wavenumber and the parameter $\sigma_{0}$ has the standard values: 0.07 for $k \leq$ $k_{p}$ and 0.09 for $k>k_{p}$. The Philips parameter $\alpha$ is related with the desired significant wave height and the peak enhancement factor $\gamma$ specifies the spectral bandwidth.

The directional spreading is taken to be of the form

$$
D(\theta)= \begin{cases}\cos ^{N}(\theta) / \Lambda(N), & |\theta| \leq \pi / 2 \\ 0, & \text { otherwise }\end{cases}
$$

where $\Lambda(N)$ is the normalizing factor of the directional distribution function and $N$ is a measure of the directional spreading, different values of which can be used, ranging from fairly long-crested (large $N$ ) to short-crested ( small $N$ ) waves. In order to be consistent with the experimental results, the following values have been selected in numerical simulations: $N$ $=840,200,90,50$ and 24, as plotted in Fig. 1. In the temporal evolution, the corresponding directional wavenumber spectrum is given by $S(\boldsymbol{k})=S(k, \theta) / k$, and the computations are initiated by specifying the spatial Fourier transform of $B$ at $t=0$,

$$
\widehat{B}\left(\boldsymbol{K}_{m n}, 0\right)=\sqrt{-2 S\left(\boldsymbol{K}_{m n}\right) \Delta K_{x} \Delta K_{y} \log \left(\hat{\theta}_{m n}\right)} \exp ^{\mathrm{i} 2 \pi \theta_{m n}},
$$

where $\hat{\theta}_{m n}$ and $\theta_{m n}$ are independently taken to be uniformly distributed on $[0,1)$, and $\boldsymbol{K}_{m n}=$ $\left(m \Delta K_{x}, n \Delta K_{y}\right)$. The physical amplitude $B\left(\boldsymbol{x}_{j k}, t\right)$ is obtained through the following discrete Fourier transform 


$$
B\left(\boldsymbol{x}_{j k}, t\right)=\sum_{m=-N_{x} / 2}^{N_{x} / 2} \sum_{n=-N_{y} / 2}^{N_{y} / 2} \widehat{B}\left(\boldsymbol{K}_{m n}, t\right) \exp ^{\mathrm{i} \boldsymbol{K}_{m n} \llbracket \boldsymbol{x}_{j k}} .
$$

With this initialization method, each Fourier coefficient can be an independent complex Gaussian variable with a variance proportional to the corresponding value of the spectrum. The preparation of initial condition in the spatial evolution is almost the same. The initial wave spectrum $S\left(\omega, k_{y}\right)$ can be obtained directly from $S(\boldsymbol{k})$ with the help of Jacobian transform, and one more condition has to be added in this case, that is, $\omega^{4}>g^{2} k_{y}^{2}$.

\subsection{Wave statistics}

In linear wave theory, the irregular waves can be represented by the sum of a large number of harmonic and statistically independent wave components. According to the central limit theorem, if the number of harmonics increases infinitely the wave surface elevation will tend to Gaussian distribution:

$$
f(\eta)=\frac{1}{\sqrt{2 \pi} \sigma} \exp \left[-\frac{\eta^{2}}{2 \sigma^{2}}\right]
$$

where $\sigma$ is the standard deviation of surface elevation $\eta$. A second-order correction to the linear wave surface was given by Tayfun [38], and further approximated by Socquet-Juglard et al. [22].

$$
f(\eta)=\frac{1-7 \sigma^{2} k_{p}^{2} / 8}{\sqrt{2 \sigma^{2} \pi\left(1+3 G+2 G^{2}\right)}} \exp \left(-\frac{G^{2}}{2 \sigma^{2} k_{p}^{2}}\right)
$$

where

$$
G=\sqrt{1+2 k_{p} \eta}-1
$$


In wave statistics, the higher-order statistical moments related with nonlinearity are usually expressed by the coefficients of skewness and kurtosis of the surface elevation, both of which will vanish for a Gaussian wave field, and have the following expressions respectively [39] [40]

$$
\begin{gathered}
\lambda_{3}=\frac{\left\langle\eta^{3}\right\rangle}{\sigma^{3}}, \\
\lambda_{4}=\frac{\left\langle\eta^{4}\right\rangle}{\sigma^{4}}-3 .
\end{gathered}
$$

\section{$3 \quad$ Facilities and Experiments}

\subsection{Wave basin and laboratory tests}

The laboratory experiments have been performed at the Marintek wave basin in Trondheim, Norway. Detailed descriptions of the facilities and experiments can be found in the earlier works [20] [31]. Here only a brief introduction is provided.

Waves are generated in a large rectangular wave basin with dimensions of $70 \mathrm{~m} \times 50 \mathrm{~m}$ as presented in Fig. 2. This basin is equipped with two sets of wavemakers. One is a double flap fixed along the $50 \mathrm{~m}$ side, which is a hydraulically operated unit for generating long-crested, regular and irregular waves. The other one used for the present study is installed along the 70 m side, consisting of altogether 144 individually computer-controlled flaps and being able to generate short-crested seas within a wide range of directional distributions of the wave energy. The water depth can also be changed by moving the bottom of the basin up or down, and was fixed at $3 \mathrm{~m}$ for the present experiments, i.e. $k_{p} h=12.07>3.14$, satisfying the deepwater condition for both cases. The absorbing sloping beach is also equipped on the 
opposite side to reduce the wave reflections.

In order to trace the evolution of the wave field, wave measurements have been mainly concentrated along the central line of the basin (see Fig. 2). The temporal variation of the surface displacement is recorded with a sampling frequency of $80 \mathrm{~Hz}$ by the wave probes uniformly deployed at $5 \mathrm{~m}$ intervals. Considering that the experiments were carried out in a finite size basin, the results may be affected by the discreteness of the facility. However, the aspect ratio of the facility can not significantly affect the wave statistics as illustrated in the work of Zhang et al. [41].

As to the initial conditions at the wave maker, the complex Fourier amplitudes are generated, each with its modulus randomly chosen from a Rayleigh distribution around the target spectrum, and random phases assumed to be uniformly distributed between 0 and $2 \pi$. In the present study, two different types of experiments are carried out, characterized by two different groups of Philips parameter $\alpha$ and peak enhancement factor $\gamma$ as listed in Table 1, where BFI is also calculated considering that this parameter contains information of both wave steepness and spectral bandwidth. In order to have enough samples to produce a significant statistical analysis, four realizations of the random sea surface for a given spectrum have been performed by using different sets of random amplitudes and phases. For each test, 20 min of wave records were collected, including the initial ramp-up time that has to be cut off in the analysis.

\subsection{Numerical simulation}

In numerical simulation, only the model based on the spatial version of MNLS equation is 
consistent with the laboratory tests, i.e., imposing the initial boundary condition at $x=0$ and obtaining the spatial evolution of the temporal wave series along the basin. However, the temporal version of MNLS equation provides the temporal evolution of an initial wave field imposed at $t=0$, and thus some problems will arise when comparing the numerical results with the laboratory experiments measured by wave gauges. In the following analysis, the comparison is based on the leading-order approximation that all properties are functions of $x$ $-c_{g} t$, where $c_{g}$ is the wave group velocity. Hence, it is assumed that the results at location $x=$ $L$ in the spatial evolution corresponds to those at time $t=2 T$ in the temporal evolution. This assumption has been simply checked in the work of Toffoli et al. [20] and will be further researched in this paper.

Simulations with the spatial MNLS equation were run up to $x=30 L$, with 9 outputs corresponding to the locations of wave gauges in the basin. Correspondingly, using the above mentioned approximation to relate the spatial and temporal evolution, the total duration of the temporal MNLS simulations has to be set equal to $t=60 T$. The output surface elevation is used to calculate the statistical properties of the wave field. To achieve statistically significant results, 10 realizations have been performed with the same input spectrum and different random amplitudes and phases [21]. Moreover, a large number of random realizations are expected to minimize the influence related to the discrepancy on initial conditions.

\section{Experimental and numerical results}

\subsection{Gaussian envelope}

Shemer and Dorfman [36] have made an experimental and numerical study for the spatial and 
temporal evolution of nonlinear wave groups in two dimensions. In order to provide an initial intuition on the properties of these two kinds of evolution, the associated numerical experiments have been further developed and performed to a much longer distance.

To the leading order, the initial surface elevation at the wavemaker has the following form:

$$
\eta(t, x=0)=a_{0} \exp \left[-\left(\frac{t}{m T_{0}}\right)^{2}\right] \cos \left(\omega_{0} t\right), \quad-10 T_{0}<t<10 T_{0},
$$

where the forcing amplitude and carrier wave period are selected in purpose such as $a_{0}=H_{s}$ I 2 and $T_{0}=T$. Note that in Figure $3, L_{0}$ is the carrier wave length calculated from the linear dispersion relation. The value of $m=3.5$ is chosen, which is sufficient to satisfy the narrow spectrum constrain for applying the MNLS equation [42]. The initial wave envelope $B(t, x=0)$ is reconstructed on the basis of the time series represented by Eq.(19). Then the wave envelope is simulated upstream by means of the spatial MNLS equation (e.g., Eq.(2)) up to a specified location. Hence the wave fields $B(x, t), \eta(x, t)$ are obtained in some domain $(x, t)$ and the initial conditions $B(x, t=0), \eta(x, t=0)$ can be formulated and used to work as the initial condition for temporal version of MNLS equation (e.g., Eq.(1)). The detailed explanation of this procedure can be found in the work of Slunyaev et al. [33] [43].

In the first row of Fig. 3, the temporal and spatial evolutions of the surface elevation in a wave group with an initial Gaussian envelope do not present any difference for the reason that the nonlinearity is weak, indicated directly by the slowly modulational process. However, in the second row, as the initial wave steepness increases, the discrepancy between temporal and spatial evolution is more and more obvious as these waves propagate downstream. Moreover, it is evident that both groups extend in the horizontal direction and the initially 
symmetric Gaussian envelope shapes gradually exhibit stronger front-tail asymmetry, with increasingly steep front and elongated tail. The crest-trough asymmetry is also notable and mainly attributed to the bound wave effects.

The difference indicated above is only based on the two-dimensional single wave group. More general three-dimensional waves with initial JONSWAP spectrum will be analysed and compared with experimental results in the following parts.

\subsection{Coefficients of skewness and kurtosis}

In this section, the coefficients of skewness and kurtosis are further researched in more general conditions. The former variable describes the vertical asymmetry of the wave profile and the latter one provides an indication on the occurrence of extreme events. From Fig. 4 to Fig. 7, the coefficients of skewness and kurtosis are plotted as a function of the dimensionless distance from the wavemaker, i.e., dividing $x$ with wavelength $L$ corresponding to the input peak period $T$. With regard to the error bars, the upper and lower error ranges of each point are equal to the mean value plus and minus its standard deviation, respectively.

It is well known that the nonlinear interaction between wave components can induce vertically asymmetric profile with higher, more peaked crest and shallower, more rounded trough. This deviation is mainly dominated by the bound wave effects, even though the dynamics of free wave weakly contribute as well [44]-[46]. The coefficient of skewness shows a slightly decreasing tendency along the wave basin, which is in part due to the spectral downshift that reduces the steepness and consequently the contribution of bound waves [20]. This trend is partially captured by the numerical simulations such as in the case 
of $N=840$ in Fig. 5. Although a little discrepancy can be observed between numerical simulations and experiments, both models have caught the fundamental characteristics of skewness. Moreover, the asymmetry of wave profile in temporal evolution is a little larger than that presented in spatial evolution in the case with initial $\mathrm{BFI}=0.70$.

Comparing with the skewness, the coefficient of kurtosis can deviate substantially from normality due to the nonlinear instability which gives rise to large amplitude waves [47]. Such a phenomenon is very pronounced in long-crested wave fields as presented in Fig. 6 and Fig. 7, where the statistical uncertainty indicated by the length of error bar is also very large. In agreement with the previous laboratory experiments [44] [48], it reaches its maximum after a fairly short evolution equivalent to about 15-20 wavelengths and then decreases towards the end of the basin with various degrees, depending on the initial BFI and directional spreading.

For a broad directional distribution, e.g., $N \leq 90$, the nonlinear instability no longer provides a significant contribution to the statistical properties of waves and thus the kurtosis only weakly deviates from Gaussian statistics, which can be captured by both numerical models although a little discrepancy still could be observed.

With regard to the long-crested waves, e.g., $N>200$, if the initial BFI is small such as Fig. 6 in this paper, the temporal evolution is almost in agreement with the spatial one and both numerical models have the ability to estimate the dynamics of mechanically generated waves reasonably well. However, as the initial BFI increases (see Fig. 7), the temporal version of MNLS equation apparently overestimates the coefficient of kurtosis (e.g., $N=840$ ) while the spatial model is still in agreement with the experimental result, except for a little overshoot at 
the initial stage, which disappears surprisingly in the unidirectional case.

\subsection{Surface elevation}

In this section, study is focused on the surface elevation which has been scaled by the standard deviation $\sigma$ of the concurrent wave series for the purpose of convenience. Both numerical and experimental probability density functions are compared with the Gaussian distribution as well as the Tayfun second-order model [38], which has been approximated in a more concise form by Socquet-Juglard et al. [22].

In the case of unidirectional waves, i.e., Fig. 8, the experimental probability density function only fits the Tayfun second-order distribution reasonably well at the first probe $(x / L$ =3.1) where the statistical properties of the wave field are dominated by the bound waves. As the waves propagate along the basin, a substantial deviation is observed on both tails of the distribution, which can be mainly attributed to the nonlinear dynamics of free wave modes. It is amazing that the lower tail of the probability density function can be described well by the normal distribution which is in agreement with the previously analysed results on the wave data of Marintek. Obviously, both versions of MNLS equation can work very well and are consistent with the experimental results in all locations regardless of the initial BFI.

With regard to the short-crested waves, e.g., Fig. 9 and Fig. 10, it is pronounced that the departure of the tail of the probability density function from the second-order model is significantly reduced. In other words, the coexistence of a number of wave components with different propagating directions leads to a huge reduction on the effects of the nonlinear instability, even in the case with a larger initial BFI. The discrepancy between spatial and 
temporal simulations can be neglected and both models are able to capture the aforementioned deviations very well even though they are not fully nonlinear.

Another very important nonlinear property of surface elevations, i.e. being positively skewed, can be observed in Fig. 11 and fitted well by the second-order model. Obviously, this phenomenon is induced by the bound wave effect. Furthermore, it is evident that the peak value of p.d.f. is larger than the second-order prediction only in the case of long-crested waves. Hence it can be inferred that this kind of deviation should mainly attribute to the nonlinear instability induced by the quasi-resonant or resonant four-wave interaction and apparently is represented very well by both temporal and spatial MNLS equations.

As shown in Figs. 12 and 13, the averaged maximal surface elevations in temporal evolution are consistent with those obtained in spatial evolution in all sea states. Comparing with the laboratory observations, the agreement is still reasonably good for unidirectional waves as indicated in Fig. 12 while the difference is evident in directional sea states as presented in Fig. 13, especially in the case with larger initial BFI $(\gamma=6)$, which is reasonable considering that the surface elevation is only measured at one point in the wave basin. Since these probes are arranged in the central line, the large discrepancy reveals that the maximal surface elevation appears outside the middle of wave basin, which may be explained by the increase of mean directional spread in the evolution process [21]. However, the averaged maximal surface elevations in three-dimensional waves are still possible to be evaluated within the framework of Piterbarg theory, explained in detail by Krogstad et al.[49] [22] [50]. Except for the case of $N=840$, the theoretical estimations (solid and dash lines) have given a good prediction on the numerical simulation results. 
It has been reported that the maximal kurtosis in directional wave field will decrease as the initial mean directional spreading increases [20], and the similar tendency is discovered for the extreme surface elevation as illustrated in Fig. 14. Thus to a certain degree, it confirms the existed theory that the coefficient of kurtosis can work as an indication on the formation of extreme crest in three-dimensional water waves [51] [52], as well as in two-dimensional case [53] . In order to compare with the ideal long-crested waves, the unidirectional case has been denoted as $N=2000$ in Fig. 14. What is worth mentioning is that the maximal extreme surface elevation appears in the case of $N=840$ rather than in the unidirectional waves in both numerical simulations and the first experimental result. As expected, the discrepancy between numerical simulations and laboratory experiments is obvious in three-dimensional sea states due to the different sizes of observed domain and individual wave, and one of the theoretical prediction methods can be found in the work of Krogstad et al.[49].

\section{Conclusions}

Based on the comparison of numerical simulations with laboratory tests in Marintek, an attempt is made to check the numerical difference between temporal and spatial versions of MNLS equation on modelling two-dimensional and three-dimensional wave fields. To trace the evolution property of an initial Gaussian surface, some statistical parameters such as the coefficients of skewness and kurtosis, the p.d.f. of the surface elevation are systemically investigated as a function of directional spreading and BFI.

For a wave group with an initial Gaussian envelope, both numerical models can catch the critical feature of gradual change of an initially symmetric shape into a strongly asymmetric 
one. In the presence of strong nonlinearity, the temporal evolution is not always in agreement with the spatial one and can present a large discrepancy in the peak stage of the evolution process, which may be partially attributed to the difference in the dispersion relation.

A slightly decreasing tendency can be observed for the coefficient of skewness in the wave basin, partially due to the spectral downshift that reduces the steepness and consequently the contribution of bound waves. Although a little discrepancy is seen between numerical simulations and experimental results, both wave models are able to catch the fundamental characteristics of asymmetry of wave profiles.

As to the coefficient of kurtosis, discrepancies are encountered in the case with narrow directional spreading and large initial BFI, performing an overestimation on the kurtosis at short distances from the wavemaker, particularly for the temporal evolution mode. However, this discrepancy is insignificant in unidirectional waves as presented in the numerical simulations governed by 2D spatial and temporal MNLS equations. For the sea states with broader directional spreading, independent of initial BFI, both numerical models estimate the evolution of this statistical variable reasonably well.

With regard to the probability density function of surface elevation, the two numerical models are capable of capturing the deviation on both tails that cannot be described by second-order theory in long-crested sea state. Furthermore, in the case of short-crested waves, the temporal and spatial MNLS simulations are in a good agreement with the statistics observed in laboratory tests, of which deviation from normal distribution is mainly attributed to the bound wave effects. Finally, considering the peak value of p.d.f. is larger than second- 
order prediction only in the case of long-crested waves, it can be concluded that this departure is mainly generated by the nonlinear instability induced by the quasi-resonant or resonant four-wave interaction.

Under most conditions, the evolution of maximal surface elevation in temporal model is consistent with that indicated in spatial model. However, the discrepancy between numerical simulations and laboratory observations are enlarged in the cases of short-wave due to different sizes of observed domain and individual wave, which may also cast some light on the location of extreme events in three-dimensional wave filed considering that these wave probes are limited and only located in the centre of wave basin.

For the selected sea states, the extreme surface elevation will increase with the decrease of initial directional spreading in three-dimensional wave field, which is in agreement with the variation tendency of the coefficient of kurtosis. However, both numerical simulations reveal that the maximal extreme surface elevation seems to appear in the case of $N=840$ rather than in the unidirectional waves.

\section{Acknowledgements}

The first author gratefully acknowledges O. Gramstad, L. Shemer and A. Slunyaev for interesting discussions and useful help in solving numerical problems related to the development of the present code of Dysthe formulation. Financial support by Portuguese Foundation for Science and Technology under the grant SFRH/BD/98983/2013 is also acknowledged. M.O. was supported by ONR Grant No. 214N000141010991 and by MIUR Grant PRIN 2012BFNWZ2. M.O. thanks Dr. B. Giulinico for fruitful discussions. 


\section{References}

[1] Kjeldsen S. Examples of heavy weather damage caused by giant waves. SNAJ Bulletin Society of Naval Architecture Japan 1997; 820: 744-748.

[2] Kharif C. Pelinovsky E. Physical mechanisms of the rogue wave phenomenon. European Journal of Mechanics B/Fluids 2003; 22: 603-634.

[3] Lighthill MJ. Contribution to the theory of waves in nonlinear dispersive systems. Journal of the Institute of Mathematics and its Applications 1965; 1: 269-306.

[4] Benjamin TB. Feir JE. The disintegration of wave trains on deep water. Part 1. Theory, Journal of Fluid Mechanics 1967; 27: 417-430.

[5] Zakharov VE. Stability of periodic waves of finite amplitude on a surface of deep fluid. Journal of Applied Mechanics and Technical Physics 1968; 9: 190-194.

[6] Yuen HC. Lake BM. Nonlinear deep water waves: theory and experiment. Physics of Fluids 1975; 18: 956-960.

[7] Shemer L. Kit E. Jiao H. Eitan O. Experiments on nonlinear wave groups in intermediate water depth. Journal of Waterway, Port, Coastal, and Ocean Engineering 1998; 124: 320327.

[8] Dysthe KB. Note on a modification to the nonlinear Schrödinger equation for application to deep water waves. Proceedings of the Royal Society of London A 1979; 369: 105-114. 
[9] Stiassnie M. Note on the modified nonlinear Schrödinger equation for deep water waves. Wave motion 1984; 6: 431-433.

[10] Trulsen K. Dysthe KB. A modified nonlinear Schrödinger equation for broader bandwidth gravity waves on deep water. Wave Motion 1996; 24: 281-289.

[11] Trulsen K. Kliakhandler I. Dysthe KB. Velarde MG. On weakly nonlinear modulation of waves on deep water. Physics of Fluids 2000; 12: 2432-2437.

[12] Lo E. Mei CC. A numerical study of water-wave modulation based on a higher-order nonlinear Schrödinger equation. Journal of Fluid Mechanics 1985; 150: 395-416.

[13] Kit E. Shemer L. Spatial versions of the Zakharov and Dysthe evolution equations for deep water gravity waves. Journal of Fluid Mechanics 2002; 450: 201-205.

[14] Annenkov SY. Shrira VI. Numerical modelling of water-wave evolution based on the Zakharov equation. Journal of Fluid Mechanics 2001; 449: 341-371.

[15] Bateman WJD. Swan C. Taylor PH. On the efficient numerical simulation of directionally spread surface water waves. Journal of Computational Physics 2001; 174: 277-305.

[16] Clamond D. Grue J. A fast method for fully nonlinear water-wave computations. Journal of Fluid Mechanics 2001; 447: 337-355.

[17] Fochesato C. Grilli S. Dias F. Numerical modelling of extreme rogue waves generated by directional energy focusing. Wave Motion 2007; 26: 395-416. 
[18] Dommermuth DG. Yue DK. A high-order spectral method for the study of nonlinear gravity waves. Journal of Fluid Mechanics 1987; 184: 267-288.

[19] West BJ. Brueckner KA. Jand RS. Milder DM. Milton RL. A new method for surface hydrodynamics. Journal of Geophysical Research 1987; 92 (C11): 11803-11824.

[20] Toffoli A. Gramstad O. Trulsen K. Monbaliu J. Bitner-Gregersen EM. Onorato M. Evolution of weakly nonlinear random directional waves: laboratory experiments and numerical simulations. Journal of Fluid Mechanics 2010; 664: 313-336.

[21] Xiao WT. Liu YM. Wu GY. Yue DKP. Rogue wave occurrence and dynamics by direct simulations of nonlinear wave-field evolution 2013; 720: 357-392.

[22] Socquet-Juglard H. Dysthe K. Trulsen K. Krogstad HE. Liu JD. Distribution of surface gravity waves during spectral changes. Journal of Fluid Mechanics 2005; 542: 195-216.

[23] Gramstad O. Trulsen K. Influence of crest and group length on the occurrence of freak waves. Journal of Fluid Mechanics 2007; 582: 463-472.

[24] Boccotti P. Wave mechanics for ocean engineering. Elsevier, Oxford 2000.

[25] Boccotti P. Wave mechanics and wave loads on marine structures. Elsevier, 2014.

[26] Petrova PG. Arena F. Guedes Soares C. Space-time evolution of random wave groups with high waves based on the quasi-determinism theory. Ocean Engineering 2011; 38 (14-15): 1640-1648. 
[27] Janssen PAEM. Nonlinear four-wave interactions and freak waves. Journal of Physical Oceanography 2003; 33: 863-884.

[28] Onorato M. Osborne AR. Serio M. Extreme wave events in directional random oceanic sea states. Physics of Fluids 2002; 14(4): 25-28.

[29] Stansberg CT. Effects from directionality and spectral bandwidth on non-linear spatial modulations of deep-water surface gravity waves. In Proceedings, Vol. 1, the $24^{\text {th }}$ International Conference on Coastal Engineering, Kobe, Japan, 1994; 579-593.

[30] Waseda T. Impact of directionality on the extreme wave occurrence in a discrete random wave system. In Proceedings of $9^{\text {th }}$ International Workshop on Wave Hindcasting and Forecasting, Victoria, Canada, 2006.

[31] Onorato M. Cavaleri L. Fouques S. Gramstad O. Janssen PAEM. Monbaliu J. Osborne AR. Pakozdi C. Serio M. Stansberg CT. Toffoli A. Trulsen K. Statistical properties of mechanically generated surface gravity waves: a laboratory experiment in a threedimensional wave basin. Journal of Fluid Mechanics 2009; 627: 235-257.

[32] Trulsen K. Stansberg CT. Spatial evolution of water surface waves: numerical simulation and experiment of bichromatic waves. Proceedings of the Eleventh (2001) International Offshore and Polar Engineering Conference, Stavanger, Norway, 2001; 7177.

[33] Slunyaev A. Pelinovsky E. Guedes Soares C. Modeling freak waves from the North Sea. Applied Ocean Research 2005; 27: 12-22. 
[34] Fedele F. Cherneva Z. Tayfun MA. Guedes Soares C. Nonlinear Schrödinger invariants and wave statistics. Physics of Fluids 2010; 22: 036601-1-036601-9, http: // dx.doi.org $/ 10.1063 / 1.3325585$.

[35] Zhang HD. Guedes Soares G. Cherneva Z. Onorato M. Modelling extreme wave heights from laboratory experiments with the nonlinear Schrödinger equation. Natural Hazards and Earth System Sciences 2013; 14: 959-968.

[36] Shemer L. Dorfman B. Experimental and numerical study of spatial and temporal evolution of nonlinear wave groups. Nonlinear Processes in Geophysics 2008; 15: 931942.

[37] Lo E. Mei CC. Slow evolution of nonlinear deep-water waves in two horizontal directions: a numerical study. Wave Motion 1987; 9: 245-259.

[38] Tayfun MA. Narrow-band nonlinear sea waves. Journal of Geophysical Research 1980; 85(C3): 1548-1552.

[39] Longuet-Higgins MS. The effect of non-linearities on statistical distribution in the theory of sea waves. Journal of Fluid Mechanics 1963; 17: 459-480.

[40] Tayfun MA. Lo J. Nonlinear effects on wave envelope and phase. Journal of Waterway, Port, Coastal, and Ocean Engineering 1990; 116: 79-100.

[41] Zhang HD. Cherneva Z. Guedes Soares C. Joint distributions of wave height and period in laboratory generated nonlinear sea states. Ocean Engineering 2013; 74: 72-80. 
[42] Shemer L. Kit E. Jiao H. An experimental and numerical study of the spatial evolution of unidirectional nonlinear water-wave groups. Physics of Fluids 2002; 14: 3380-3390.

[43] Slunyaev A. Pelinovsky E. Guedes Soares C. Reconstruction of extreme events through numerical simulations. Journal of Offshore Mechanics and Arctic Engineering 2014; 136: 011302-1-011302-10.

[44] Onorato M. Osborne AR. Serio M. Cavaleri L. Modulational instability and nonGaussian statistics in experimental random water-wave trains. Physics of Fluids 2005; 17: 078101.

[45] Mori N. Onorato M. Janssen PA. Osborne AR. Serio M. On the extreme statistics of long-crested deep water waves: Theory and experiments. Journal of Geophysical Research 2007; 112(C09011): doi:10.1029/2006JC004024.

[46] Toffoli A. Onorato M. Bitner-Gregersen EM. Osborne AR. Babanin AV. Surface gravity waves from direct numerical simulations of the Euler equations: A comparison with second-order theory. Ocean Engineering 2008; 35: 367-379.

[47] Onorato M. Osborne A. Serio M. Cavaleri L. Brandini C. Stansberg C. Extreme waves, modulational instability and second order theory: wave flume experiments on irregular waves. European Journal of Mechanics B/ Fluids 2006; 25: 586-601.

[48] Zhang HD. Cherneva Z. Guedes Soares C. Onorato M. Comparison of distributions of wave heights from nonlinear Schrödinger equation simulations and laboratory 
experiments. Journal of Offshore Mechanics and Arctic Engineering 2015; 137(5): doi: 10.1115/1.4031218.

[49] Krogstad HE. Liu JD. Socquet-Juglard H. Dysthe KB. Trulsen K. Spatial extreme value analysis of nonlinear simulations of random surface waves. Proceedings of the 23rd International Conference on Ocean, Offshore and Arctic Engineering, Vancouver, Canada, 2004; OMAE 2004-51336.

[50] Dysthe K. Socquet-Juglard H. Trulsen K. Krogstad HE. Liu JD. "Freak" waves and large-scale simulations of surface gravity waves. Rogue Waves, Proceedings of the 14th Aha Huliko'a Hawaiian Winter Workshop, 2005; 91-99.

[51] Guedes Soares C. Cherneva Z. Antão E. Characteristics of abnormal waves in North Sea storm sea states. Applied Ocean Research 2003; 25: 337-344.

[52] Guedes Soares C. Cherneva Z. Antão E. Abnormal waves during the hurricane Camille. Journal of Geophysical Research 2004; 109, C08008.

[53] Zhang HD. Guedes Soares C. and Onorato M. Modelling of the Spatial Evolution of Extreme Laboratory Wave Crest and Trough Heights with the NLS-Type Equations. Applied Ocean Research 2015; 52, 140-150. 
Table 1. Parameters in the experiments.

\begin{tabular}{ccccccc}
\hline Case & $\alpha$ & $\gamma$ & $H_{s}(\mathrm{~m})$ & $T(\mathrm{~s})$ & $k_{p} H_{s} / 2$ & BFI \\
\hline A & 0.014 & 3.0 & 0.06 & 1.0 & 0.13 & 0.70 \\
B & 0.016 & 6.0 & 0.08 & 1.0 & 0.16 & 1.10 \\
\hline
\end{tabular}

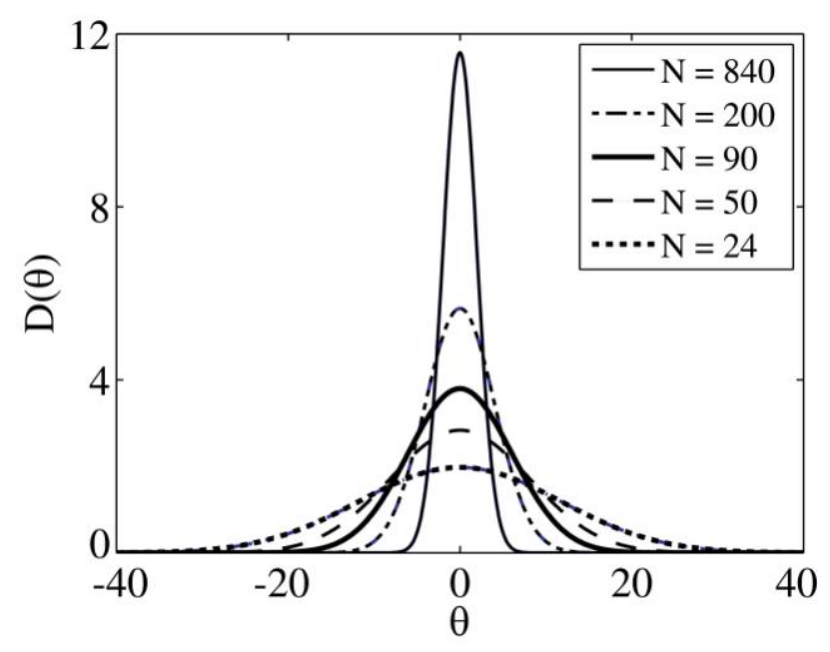

Fig. 1. Angular distributions of the initial spectra.

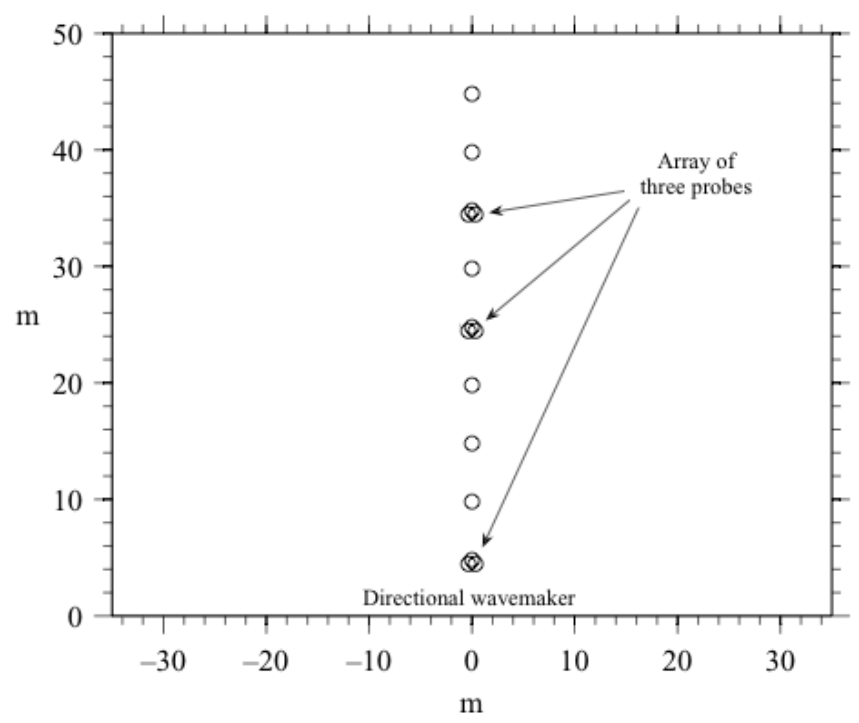

Fig. 2. Layout of wave basin. 

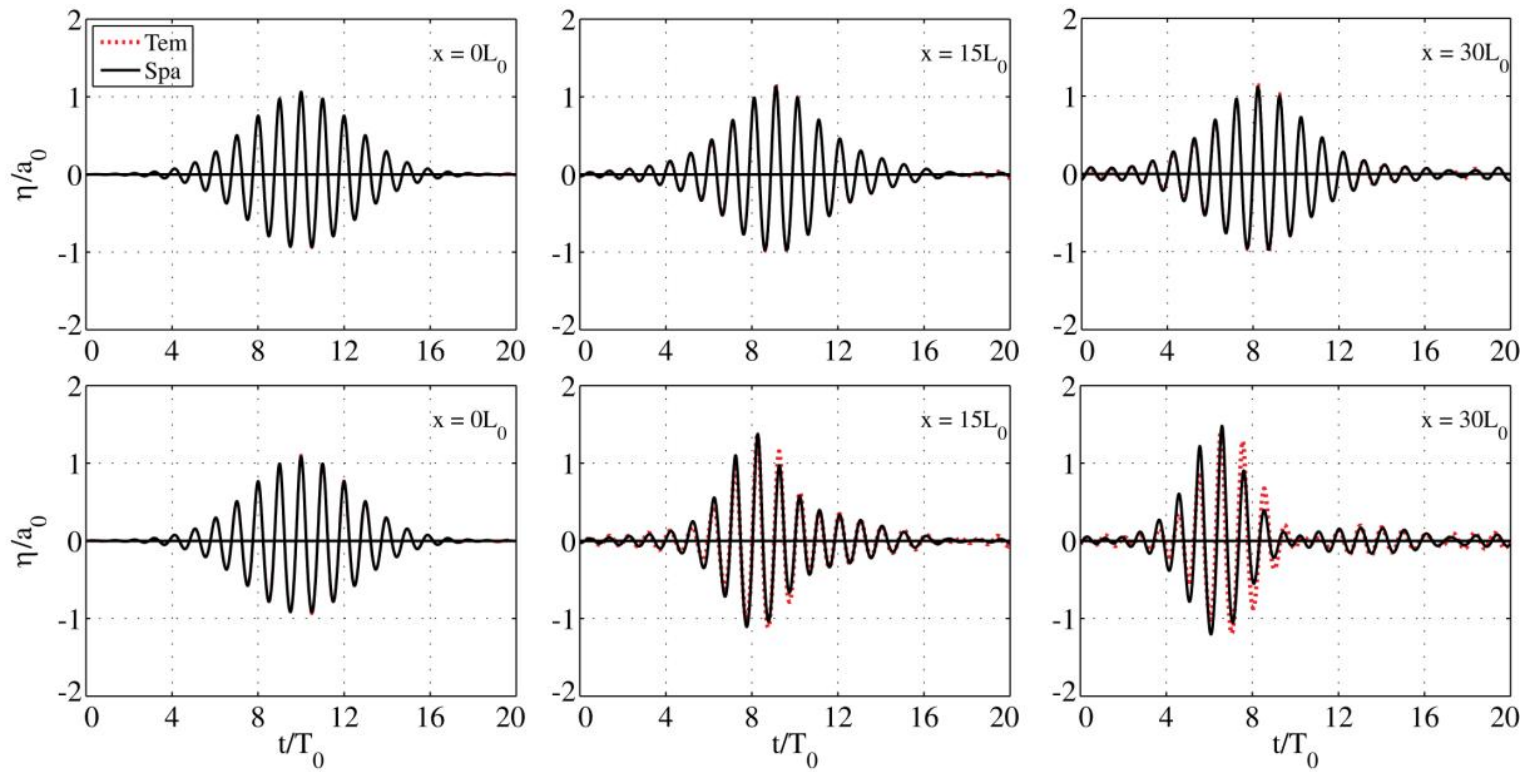

Fig. 3. Temporal variations of the surface elevation within the group obtained at different locations simulated by the spatial evolution model (black solid line), comparing with those indirectly obtained from the temporal evolution model (red dotted line). $a_{0} k_{0}=0.13$, first panel, and $a_{0} k_{0}=0.16$, second panel.

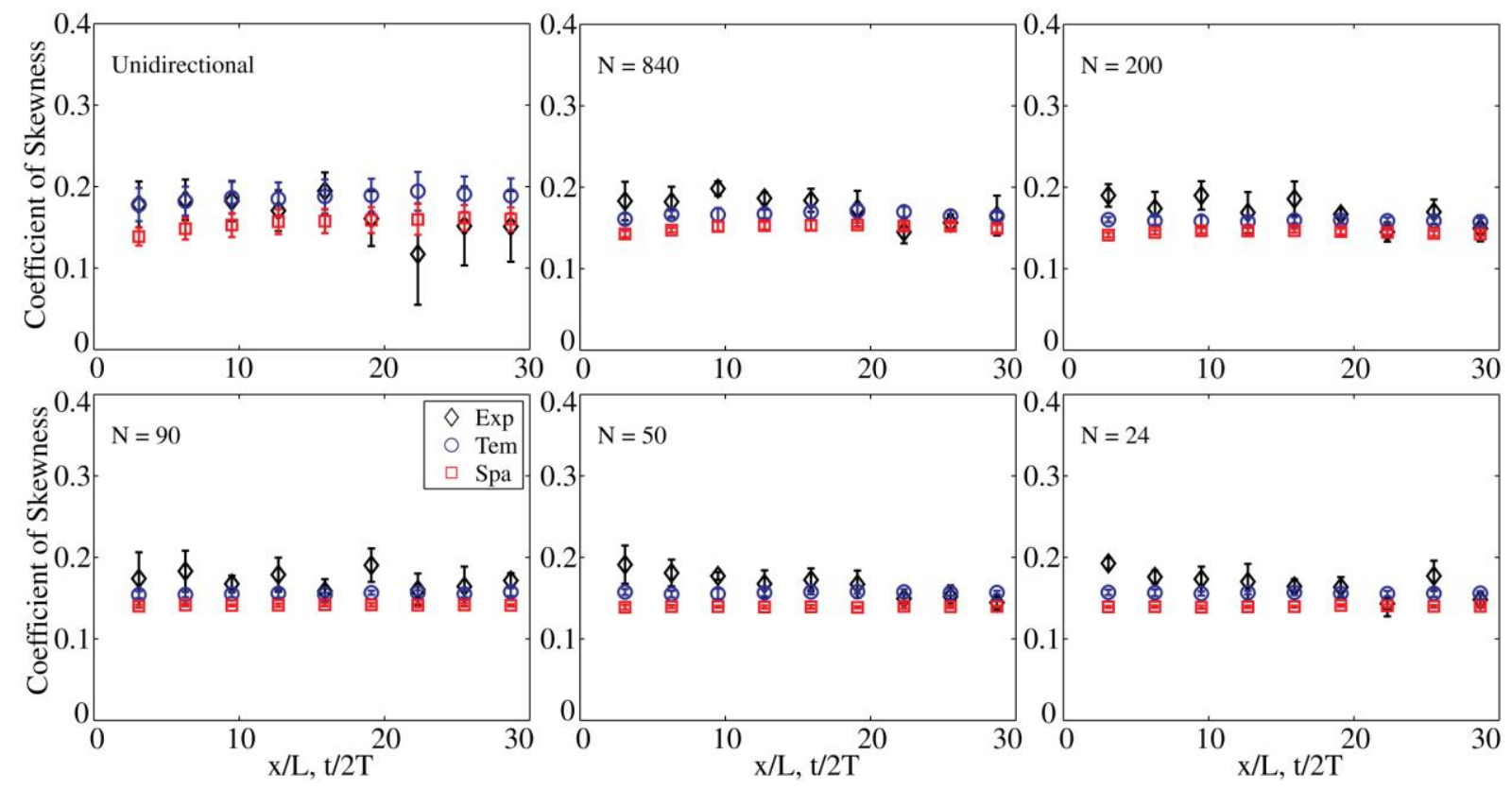

Fig. 4. Evolution of the coefficient of skewness for $\mathrm{BFI}=0.70$. 

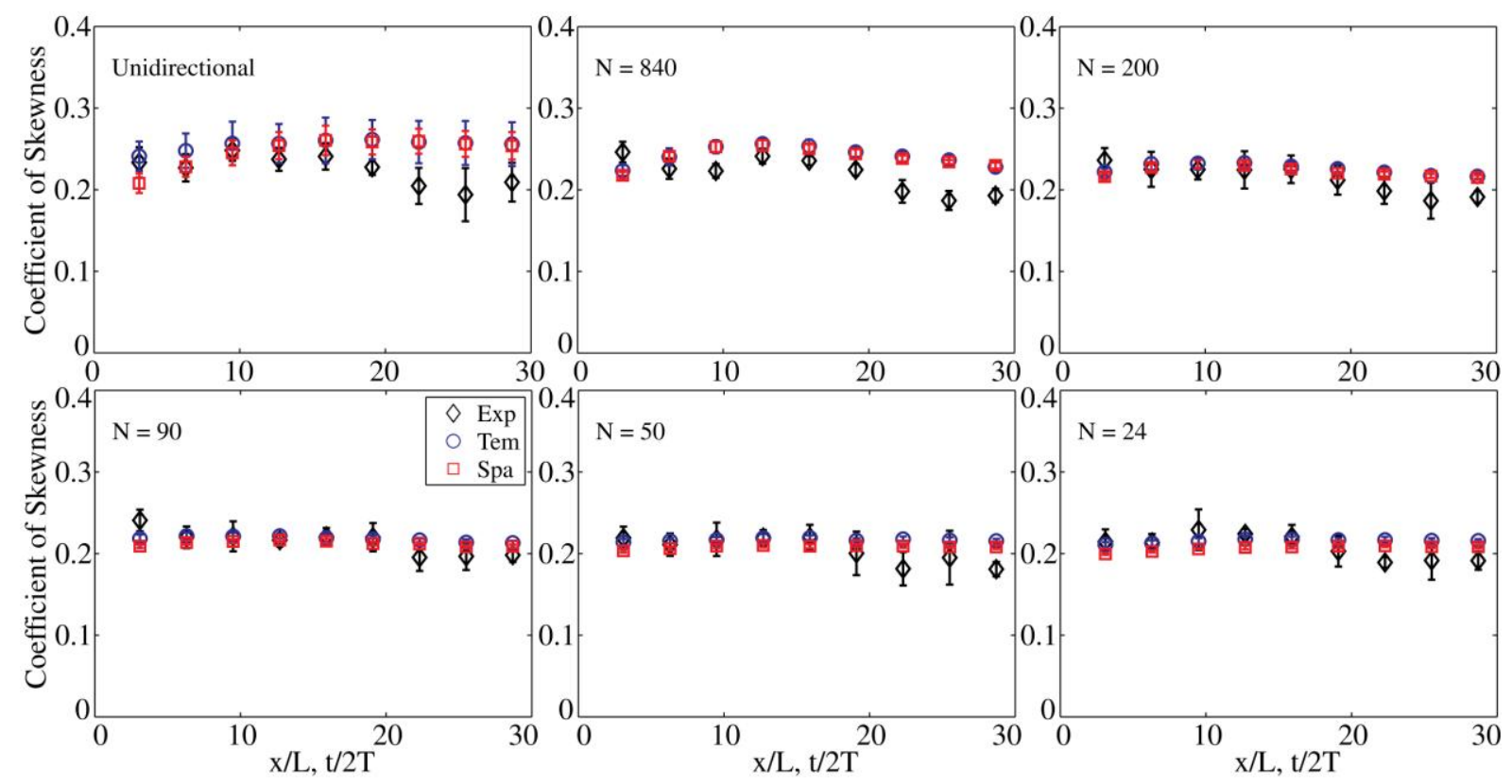

Fig. 5. Evolution of the coefficient of skewness for BFI=1.10.
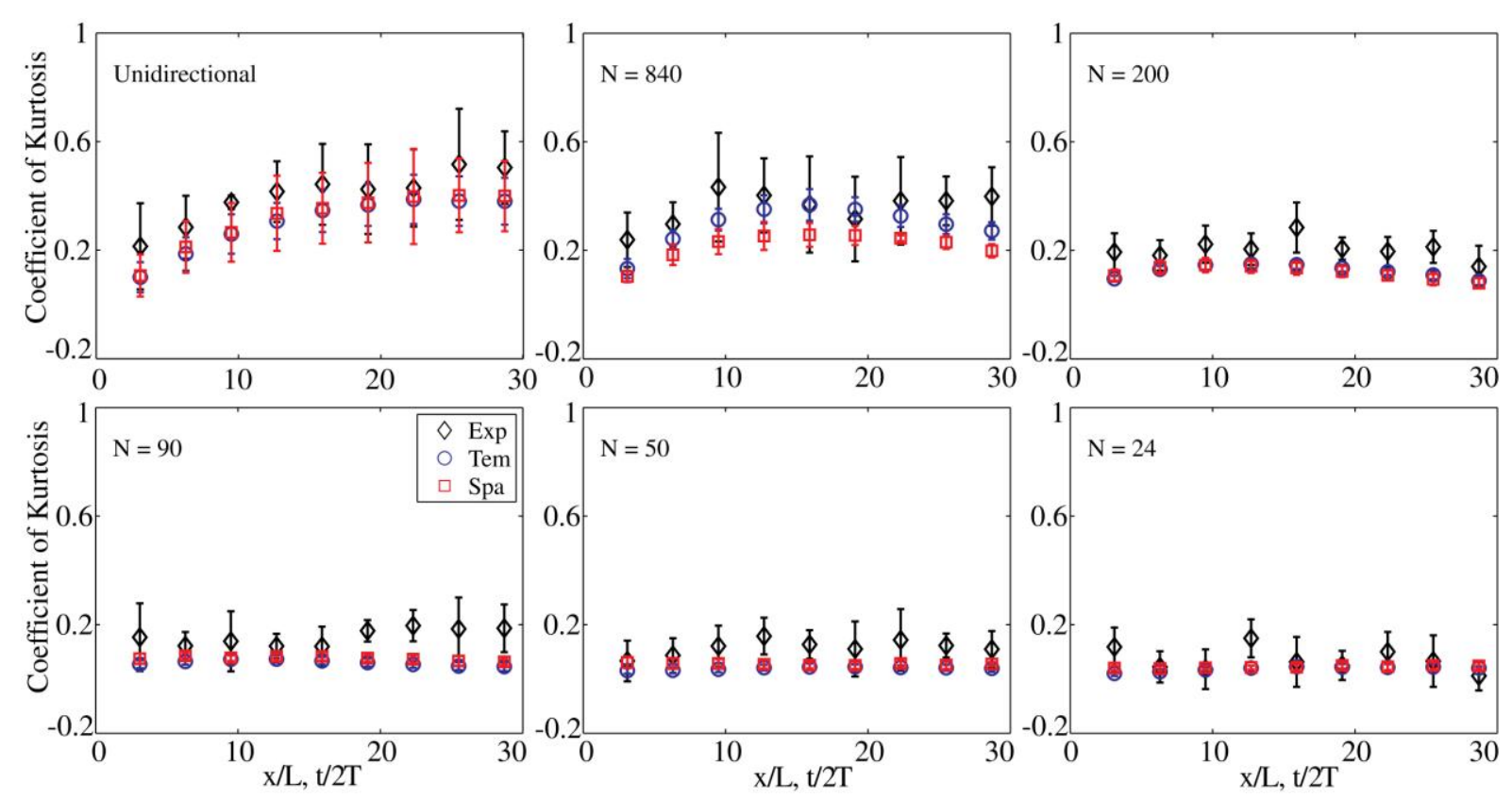

Fig. 6. Evolution of the coefficient of kurtosis for $\mathrm{BFI}=0.70$. 

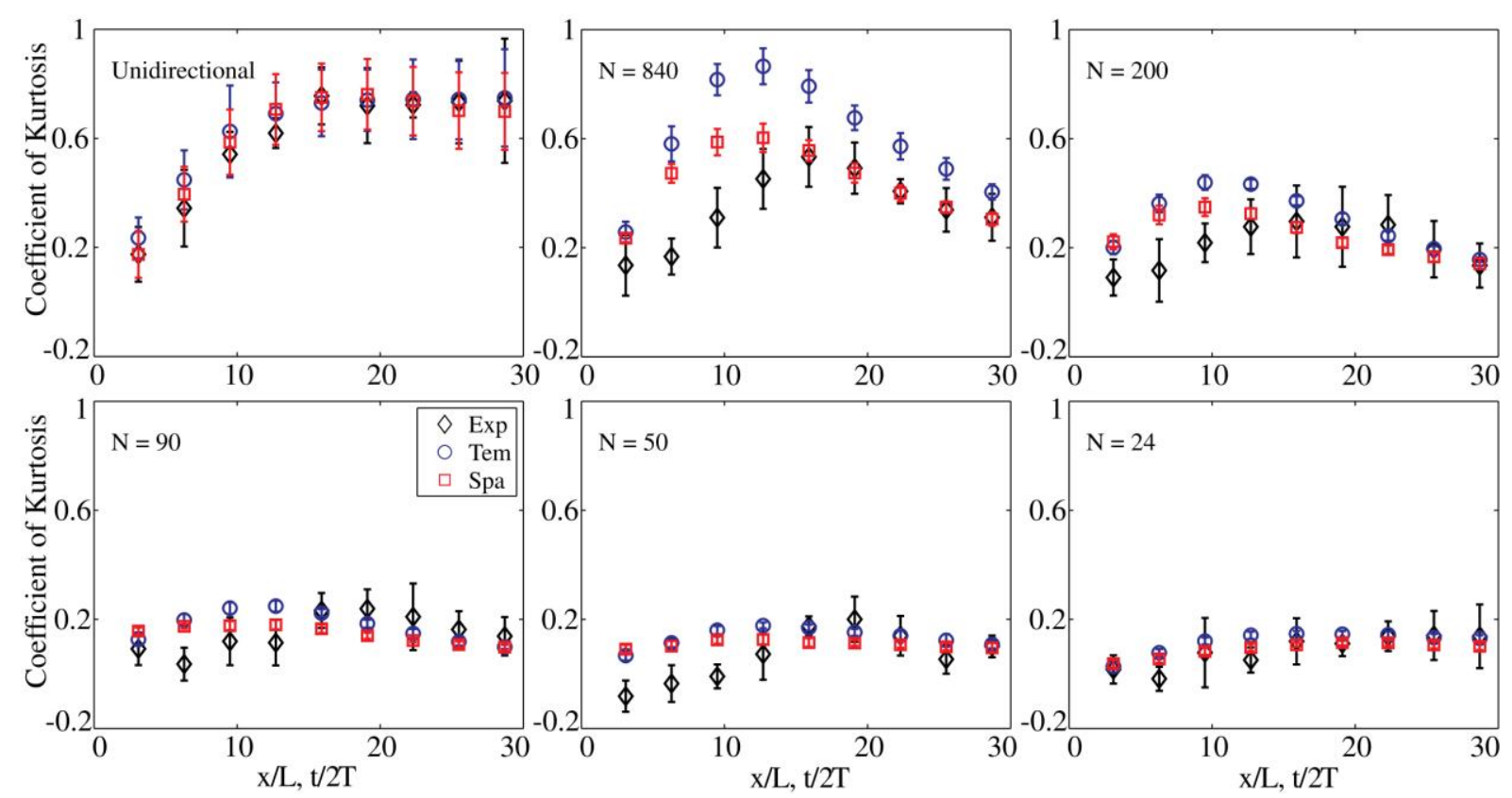

Fig. 7. Evolution of the coefficient of kurtosis for BFI=1.10.
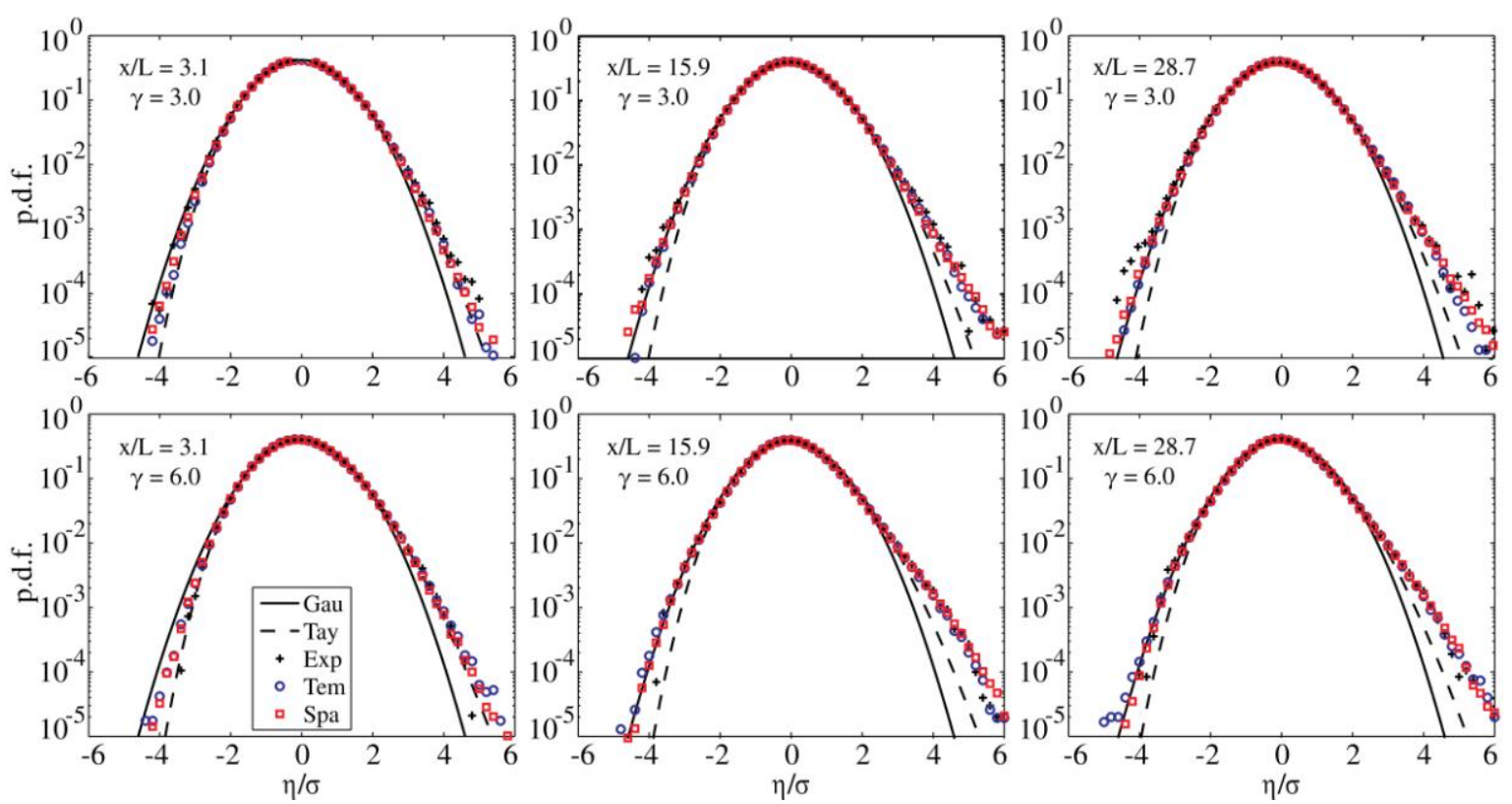

Fig. 8. Probability density function of the surface elevation for unidirectional waves. 

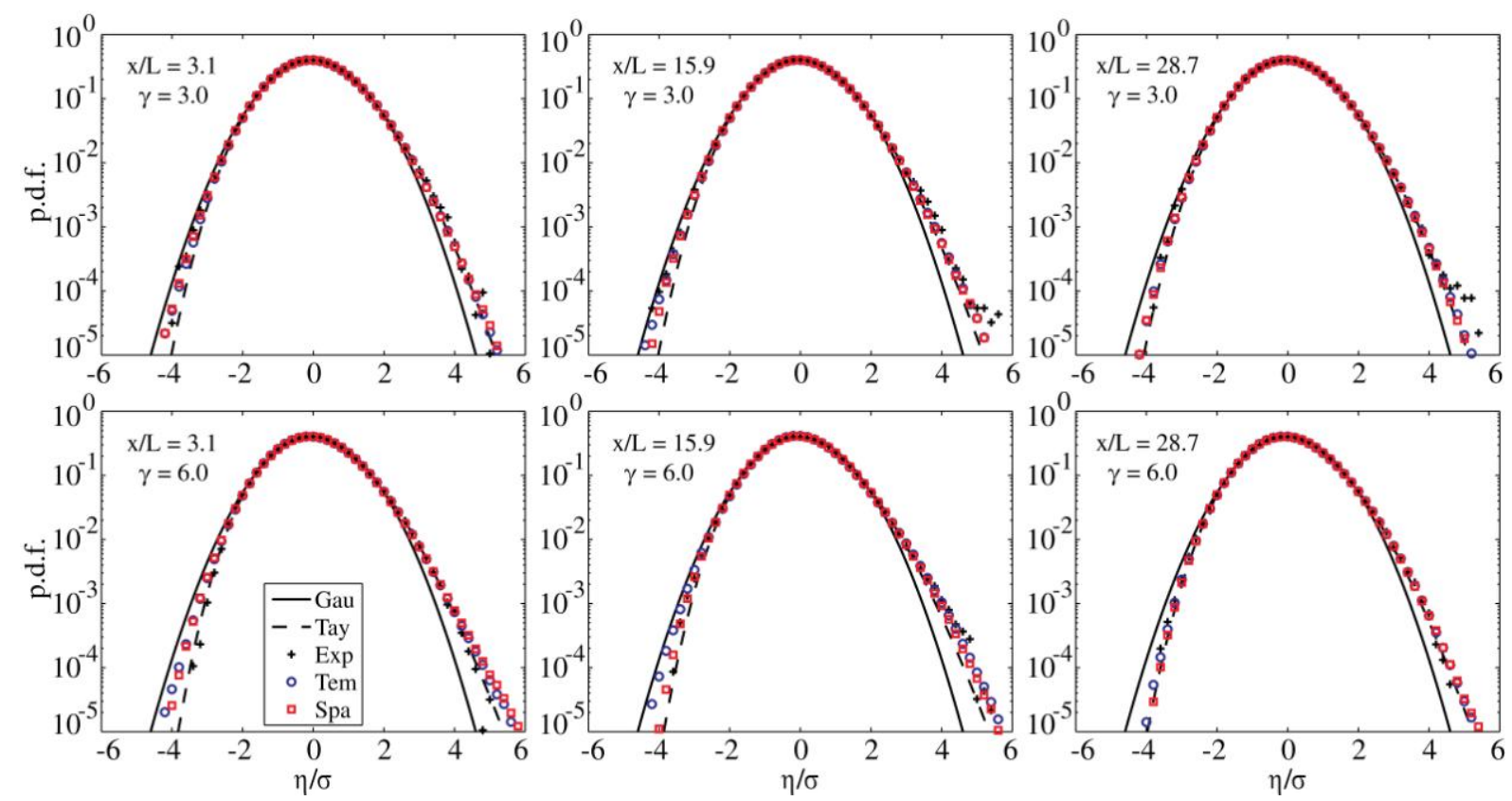

Fig. 9. Probability density function of the surface elevation with initial directional spreading $N=200$.
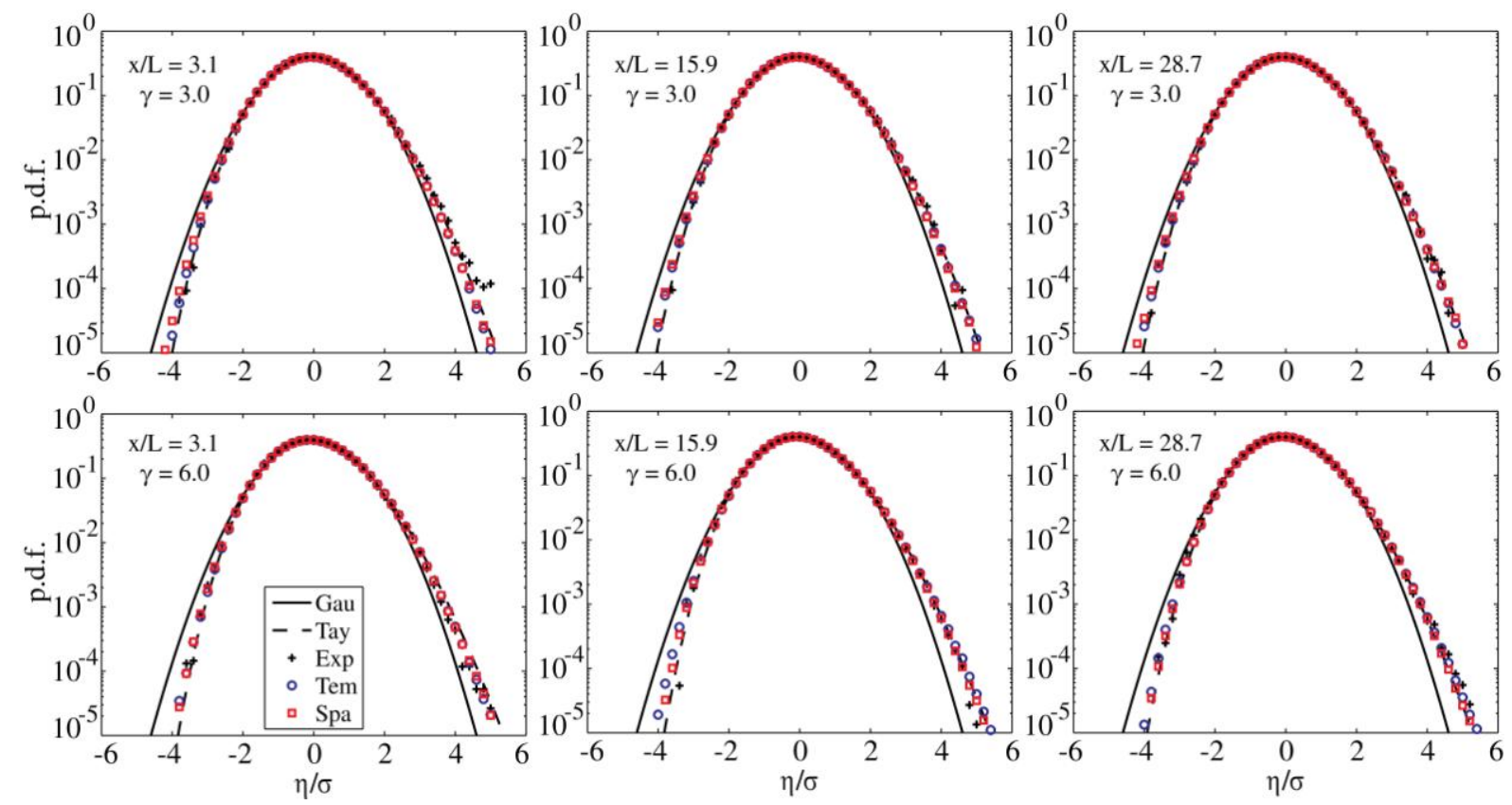

Fig. 10. Probability density function of the surface elevation with initial directional spreading $N=24$. 

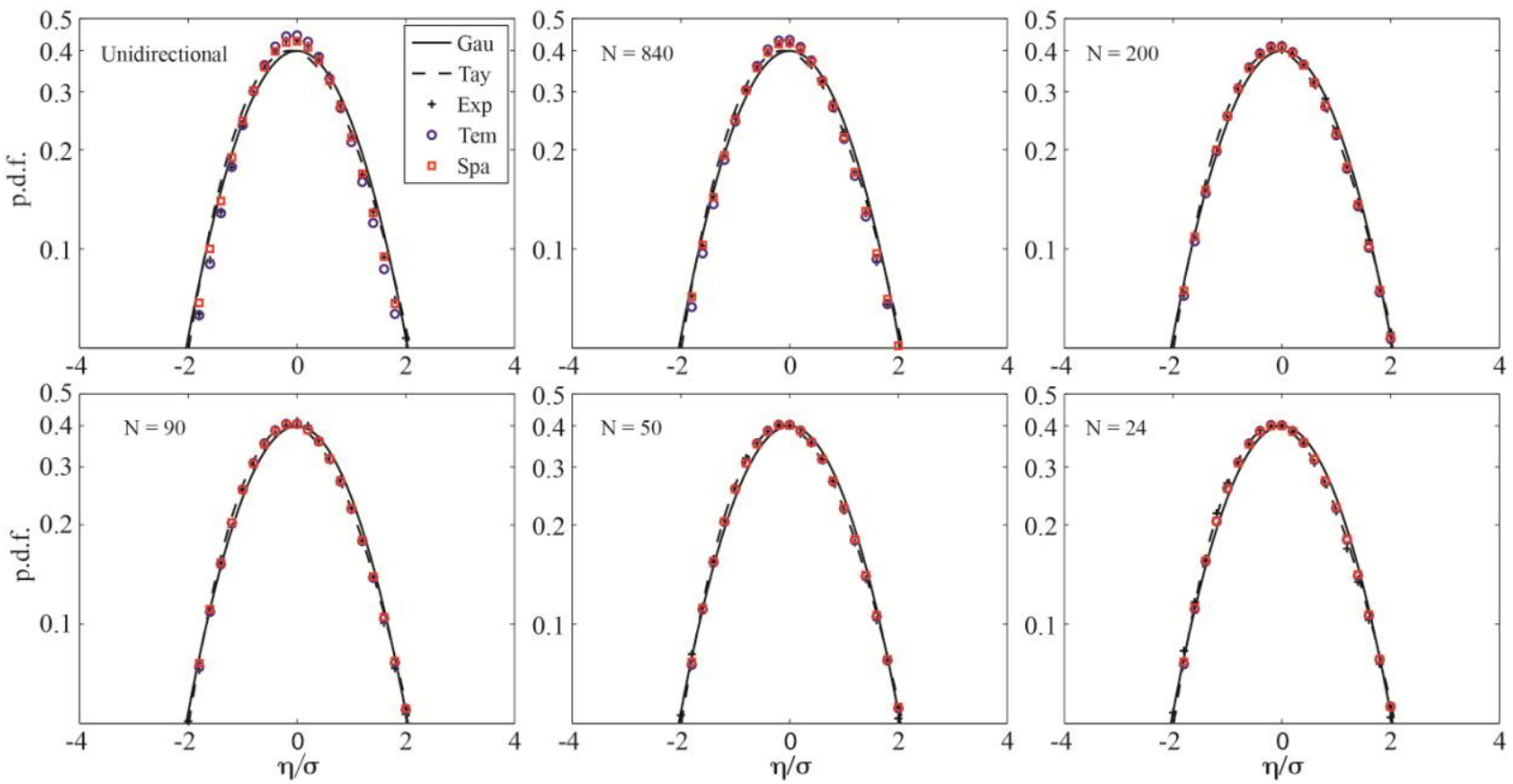

Fig. 11. Probability density function of the surface elevation located at $x / L=15.9$ with the same $\gamma=6$ and different initial directional spreading $N$.
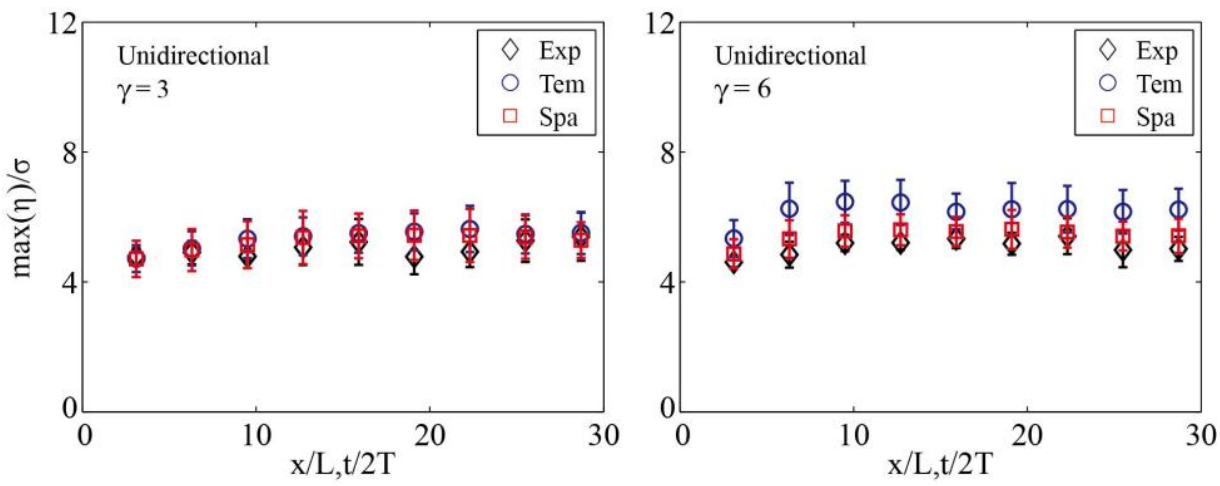

Fig. 12. Evolution of the maximal surface elevation in unidirectional sea state.
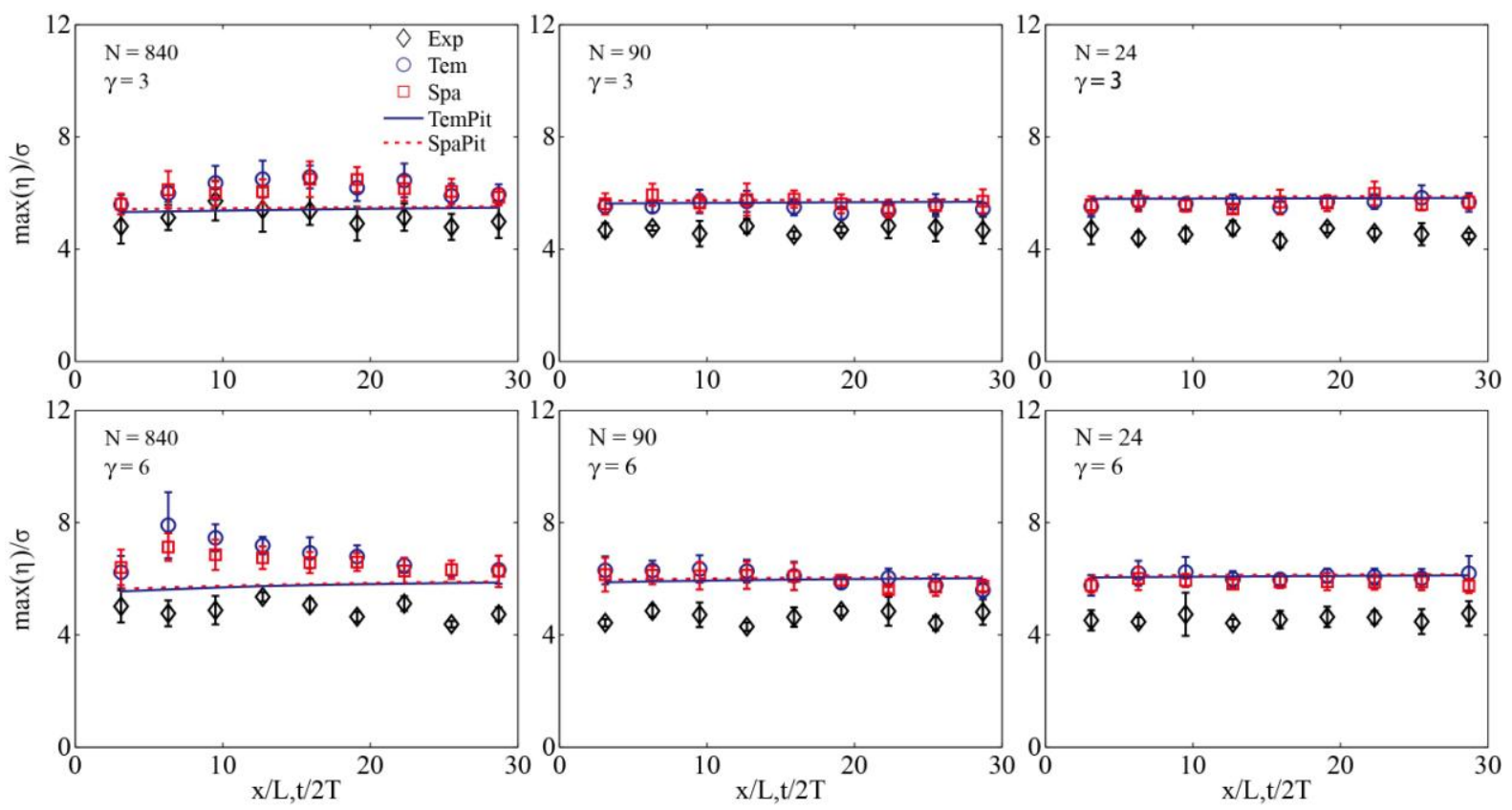

Fig. 13. Evolution of the maximal surface elevation with different initial directional spreading $N$, compared with 
the expected value given by Piterbarg-Tayfun distribution (solid and dash lines).
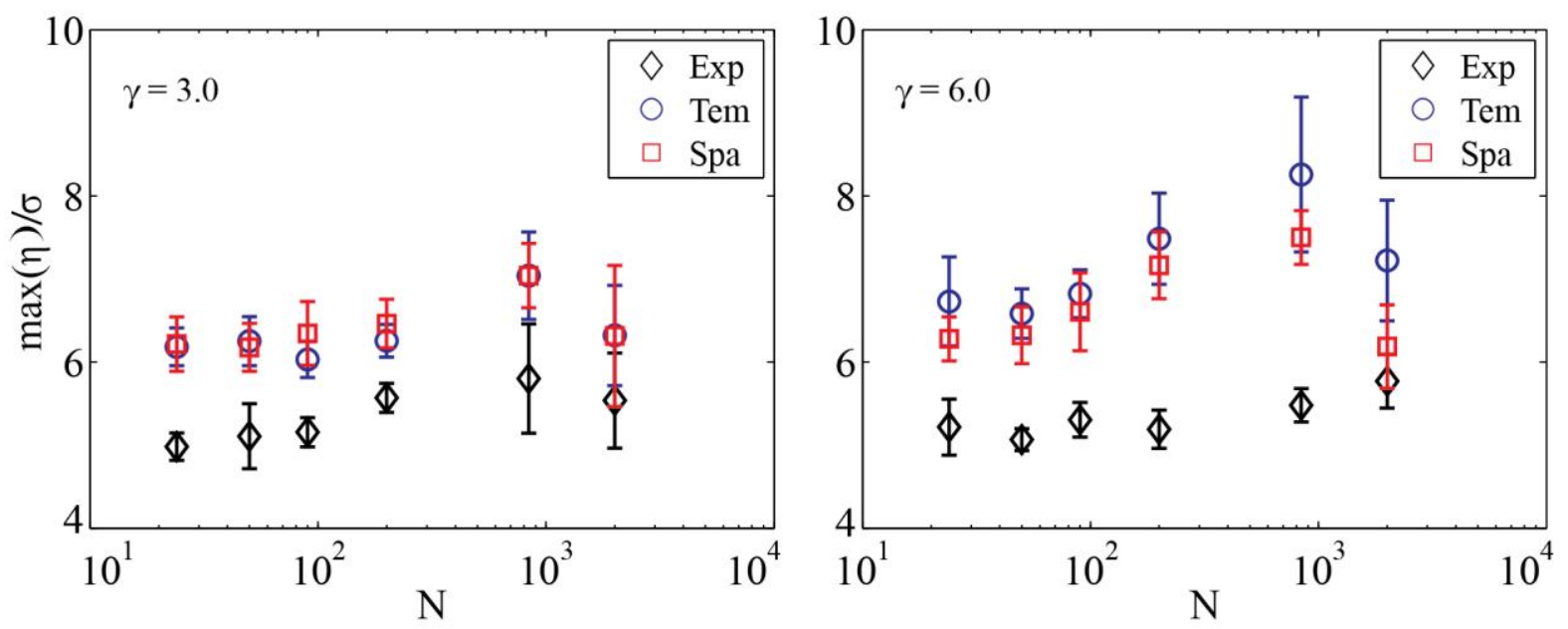

Fig. 14. Extreme surface elevation as a function of initial directional spreading $N$. Unidirectional wave is denoted as 2000 for comparison. 